\title{
Cryptanalysis of Achterbahn-128/80 with a new keystream limitation
}

\author{
María Naya-Plasencia ${ }^{\star}$ \\ INRIA, projet CODES, Domaine de Voluceau \\ 78153 Le Chesnay Cedex, FRANCE \\ Maria.Naya_Plasencia@inria.fr
}

\begin{abstract}
This paper presents two key-recovery attacks against the last modification to Achterbahn-128/80 proposed by the authors at SASC 2007 due to the previous attacks. The 80-bit variant, Achterbahn-80, has been limited to produce at most $2^{52}$ bits of keystream with the same pair of key and IV, while Achterbahn- 128 is limited to $2^{56}$. The attack against Achterbahn- 80 has complexity $2^{67}$ and needs fewer than $2^{52}$ bits of keystream, and the one against Achterbahn-128 has complexity $2^{104}$ and needs fewer than $2^{56}$ keystream bits. These attacks are based on the previous ones. The attack against Achterbahn-80 uses a new idea which provides a trade-off between the keystream length and the computational complexity when decimating.
\end{abstract}

Keywords: eSTREAM, stream cipher, Achterbahn, cryptanalysis, correlation attack, linear approximation, parity check, key-recovery attack.

\section{Introduction}

Achterbahn $[2,4]$ is a stream cipher proposal submitted to the eSTREAM project. After the cryptanalysis of the first two versions [8,7], it has moved on to a new one called Achterbahn-128/80 [3] published in June 2006. Achterbahn-128/80 corresponds to two keystream generators with key sizes of 128 bits and 80 bits, respectively. Their maximal keystream length was limited to $2^{63}$.

As this version has been attacked [6,9], the authors have proposed a new limitation of the keystream length [5], which is $2^{52}$ for Achterbahn- 80 and $2^{56}$ for Achterbahn-128. We present here two attacks against both generators, which are based on the previous ones. The attack against the 80-bit variant, Achterbahn80 , has complexity $2^{67}$ and needs fewer than $2^{52}$ keystream bits. The attack against Achterbahn-128 requires $2^{104}$ operations and fewer than $2^{56}$ keystream bits.

\footnotetext{
* This work was supported in part by the European Commission through the IST Programme under Contract IST-2002-507932 ECRYPT. The information in this document reflects only the author's views, is provided as is and no warranty is given that the information is fit for any particular purpose. The user thereof uses the information at its sole risk and liability.
} 


\section{Distinguishing attack against Achterbahn-80}

Now, we describe a new attack against Achterbahn- 80 with a complexity of $2^{67}$ where a linear approximation of the output function is considered. The attack is a distinguishing attack but it also allows to recover the initial states of certain constituent registers.

This attack is very similar to the previous attack against Achterbahn- 80 .

Our attack exploits the following linear approximation of the combining function $G$ :

$$
\ell\left(x_{1}, \ldots, x_{11}\right)=x_{1}+x_{3}+x_{4}+x_{5}+x_{6}+x_{7}+x_{10} .
$$

Since $G$ is 6-resilient, $\ell$ is the best approximation by a 7 -variable function.

For $\ell(t)=x_{1}(t)+x_{3}(t)+x_{4}(t)+x_{5}(t)+x_{6}(t)+x_{7}(t)+x_{10}(t)$, the keystream $(S(t))_{t \geq 0}$ satisfies $\operatorname{Pr}[S(t)=\ell(t)]=\frac{1}{2}\left(1-2^{-3}\right)$.

Parity-checks. Let us build a parity-check as follows:

$$
\ell \ell(t)=\ell(t)+\ell\left(t+T_{3} T_{7}\right)+\ell\left(t+T_{4} T_{5}\right)+\ell\left(t+T_{3} T_{7}+T_{4} T_{5}\right) .
$$

The terms containing the sequences $x_{3}, x_{4}, x_{5}, x_{7}$ vanish in $\ell \ell(t)$, so $\ell \ell(t)$ depends exclusively on the sequences $x_{1}, x_{6}$ and $x_{10}$. The period $T_{4} T_{5}$ is $2^{51}$ and the period $T_{3} T_{7}$ is smaller than $2^{49}$ as $T_{3}$ and $T_{7}$ have common factors, so to build those parity checks we need less than the maximal keystream length allowed.

Adding four times the approximation has the effect of multiplying the bias four times, so the bias of

$$
\sigma(t)=S(t)+S\left(t+T_{7} T_{3}\right)+S\left(t+T_{4} T_{5}\right)+S\left(t+T_{7} T_{3}+T_{4} T_{5}\right)
$$

where $(S(t))_{t \geq 0}$ is the keystream, is $2^{-4 \times 3}$.

We now decimate $\sigma(t)$ by the period of the register 1 , which is involved in the parity-check, so we create like this a new parity-check:

$$
\sigma^{\prime}(t)=\sigma\left(t\left(2^{22}-1\right)\right) .
$$

Then, if we did as in the previous attack and we performed an exhaustive search for the initial states of registers 6 and 10, we would need $2^{3 \times 4 \times 2} \times 2 \times(58-2) \times$ $\ln (2)=2^{30.29}$ parity-checks $\sigma^{\prime}(t)$ to detect this bias. As we are decimating by the period of the register 1 , we would need $2^{30.29} \times 2^{22}=2^{52.29}$ keystream bits to perform the attack, and it is over the limitation, so we cannot do that.

What we are going to do, instead of taking only the first bit of the keystream and of decimating by the period of the first register $2^{30.29}$ times, consists in considering the first four consecutive shifts of the keystream and for each one, we are going to obtain a sequence of $\frac{2^{30.29}}{4}=2^{28.29}$ bits by decimating it by the period of the first register $2^{28.29}$ times. Thus, we consider the first $2^{50.29}$ bits of the keystream and we compute the $4 \times 2^{28.29}=2^{30.29}$ parity checks: $S\left(t\left(2^{22}-1\right)+i\right)+S\left(t\left(2^{22}-1\right)+i+T_{7} T_{3}\right)+S\left(t\left(2^{22}-1\right)+i+T_{4} T_{5}\right)+$ 
$S\left(t\left(2^{22}-1\right)+i+T_{7} T_{3}+T_{4} T_{5}\right)$

for $i \in\{0, \ldots, 3\}$ and $0 \leq t<2^{28.29}$. This way, the number of keystream bits that we need is reduced to $2^{28.29} \times 2^{22}=2^{50.29}$ and respects the maximal keystream length permitted. This is going to change time complexity as now we will have to perform an exhaustive search on the four following bits generated by register 1 :

$$
z_{i}=x_{1}(i)+x_{1}\left(i+T_{7} T_{3}\right)+x_{1}\left(i+T_{4} T_{5}\right)+x_{1}\left(i+T_{7} T_{3}+T_{4} T_{5}\right)
$$

for $i \in\{0, \ldots, 3\}$. As we do not care about the real value of those bits but of the differences $\left(z_{i}+z_{0}\right) \forall i \in\{1,2,3\}$, we are going to evaluate the $2^{3}$ possible states only. Thus, we perform an exhaustive search over the registers 6 and 10, adapting to our new situation the algorithm introduced in [9]. We will have to compute, for each one of the previously mentioned sequences, so for each $i \in\{0,1,2,3\}$, the following sum:

$$
\begin{aligned}
& \sum_{t^{\prime}=0}^{2^{28.29}-1} \sigma\left(t^{\prime} T_{1}+i\right) \oplus \ell \ell\left(t^{\prime} T_{1}+i\right) \\
= & \sum_{k=0}^{T^{\prime}} \sum_{t=0}^{2} \sigma\left(\left(T_{6} t+k\right) T_{1}+i\right) \oplus \ell \ell\left(\left(T_{6} t+k\right) T_{1}+i\right) \\
+ & \sum_{k=T^{\prime}+1}^{T_{6}-1} \sum_{t=0}^{1} \sigma\left(\left(T_{6} t+k\right) T_{1}+i\right) \oplus \ell \ell\left(\left(T_{6} t+k\right) T_{1}+i\right) \\
= & \sum_{k=0}^{T^{\prime}} \sum_{t=0}^{2} \sigma\left(\left(T_{6} t+k\right) T_{1}+i\right) \oplus \sigma_{10}\left(\left(T_{6} t+k\right) T_{1}+i\right) \oplus \sigma_{6}\left(\left(T_{6} t+k\right) T_{1}+i\right) \\
+ & \sum_{k=T^{\prime}+1}^{T_{6}-1} \sum_{t=0}^{1} \sigma\left(\left(T_{6} t+k\right) T_{1}+i\right) \oplus \sigma_{10}\left(\left(T_{6} t+k\right) T_{1}+i\right) \oplus \sigma_{6}\left(\left(T_{6} t+k\right) T_{1}+i\right) \\
= & \sum_{k=0}^{T^{\prime}}\left[\left(\sigma_{6}\left(k T_{1}+i\right) \oplus 1\right)\left(\sum_{t=0}^{2} \sigma\left(\left(T_{6} t+k\right) T_{1}+i\right) \oplus \sigma_{10}\left(\left(T_{6} t+k\right) T_{1}+i\right)\right)\right. \\
+ & \left.\sigma_{6}\left(k T_{1}+i\right)\left(3-\sum_{t=0}^{2} \sigma\left(\left(T_{6} t+k\right) T_{1}+i\right) \oplus \sigma_{10}\left(\left(T_{6} t+k\right) T_{1}+i\right)\right)\right] \\
+ & \sum_{k=T^{\prime}}^{T_{6}-1}\left[\left(\sigma_{6}\left(k T_{1}+i\right) \oplus 1\right)\left(\sum_{t=0}^{1} \sigma\left(\left(T_{6} t+k\right) T_{1}+i\right) \oplus \sigma_{10}\left(\left(T_{6} t+k\right) T_{1}+i\right)\right)\right. \\
+ & \left.\sigma_{6}\left(k T_{1}+i\right)\left(2-\sum_{t=0}^{1} \sigma\left(\left(T_{6} t+k\right) T_{1}+i\right) \oplus \sigma_{10}\left(\left(T_{6} t+k\right) T_{1}+i\right)\right)\right],
\end{aligned}
$$

where $\sigma(t), \sigma_{6}(t)$ and $\sigma_{10}(t)$ are the parity checks computed at the instant $t$ with the keystream, the sequence generated by the register 6 and the one generated 
by the register 10 respectively, and where

$$
T^{\prime}=2^{28.29}-2 \times T_{6}=2^{28.29}-2^{28}+2=2^{25.83} .
$$

The sum can be written the above way since $\sigma_{6}\left(\left(T_{6} t+k\right) T_{1}+i\right)$ is constant for fixed values of $k$ and $i$. We are going to see what we have got to do at this point:

- We choose an initial state for register 6, e.g. the all-one initial state. We compute and save a binary vector $V_{6}$ of length $T_{6}$ :

$$
V_{6}[k]=\sigma_{6}(k),
$$

where the sequence with whom we are computing $\sigma_{6}(k)$ is generated from the chosen initial state. The complexity of this state is $T_{6} \times 2^{2}$ operations.

- For each possible initial state of register 10 (so $2^{31-1}$ possibilities):

- we compute and save four vectors $V_{10, i}$, where $i \in\{0,1,2,3\}$, each one composed of $T_{6}$ integers of 2 bits.

$$
V_{10, i}[k]=\sum_{t=0}^{m} \sigma\left(\left(T_{6} t+k\right) T_{1}+i\right) \oplus \sigma_{10}\left(\left(T_{6} t+k\right) T_{1}+i\right),
$$

where $m=2$ if $k \leq T^{\prime}$ and $m=1$ if $k>T^{\prime}$. The complexity of this state is:

$$
\begin{aligned}
2^{2} \times\left(3 \times 2^{25.83}+2 \times\left(2^{27}-1-2^{25.83}\right)\right) \times\left(2^{3}+2\right) & =2^{2} \times 2^{28.29} \times 2^{3.3} \\
& =2^{33.69}
\end{aligned}
$$

for each possible initial state of register 10 , where $2^{2}$ is the number of vectors that we are computing, $2^{3}$ corresponds to the number of operations required for computing each $\left(\sigma(t)+\sigma_{10}(t)\right)$ and $2^{28.29} \times 2$ is the cost of summing up $2^{28.29}$ integers of 2 bits.

- For each possible $p$ from 0 to $T_{6}-1$ :

* we define $V_{6, i}$ of length $T_{6}, \forall i \in\{0,1,2,3\}$ :

$$
V_{6, i}[k]=V_{6}\left[k+p+i \bmod T_{6}\right] .
$$

Actually, $\left(V_{6, i}[k]\right)_{k<T_{6}}$ corresponds to $\left(\sigma_{6}(k)\right)_{k<T_{6}}$ when the initial state of register 6 corresponds to the internal state obtained after clocking $(i+p)$ times register 6 from the all-one initial state.

* With the eight vectors that we have obtained $\left(V_{10,0}, \ldots, V_{10,3}, V_{6,0}, \ldots, V_{6,3}\right)$, we compute for each $i \in\{0,1,2,3\}$ :

$$
\begin{aligned}
W_{i}= & \sum_{k=0}^{T^{\prime}}\left[\left(V_{6, i}[k] \oplus 1\right) V_{10, i}[k]+V_{6, i}[k]\left(3-V_{10, i}[k]\right)\right]+ \\
& \sum_{k=T^{\prime}+1}^{T_{6}-1}\left[\left(V_{6, i}[k] \oplus 1\right) V_{10, i}[k]+V_{6, i}[k]\left(2-V_{10, i}[k]\right)\right] .
\end{aligned}
$$


Now, we do the sum of the four of them, so for all the $2^{3}$ possible states of the bits $z_{0}, \ldots, z_{3}$ coming from register 1 , and considering $z_{0}$ as positive:

$$
W_{0} \pm W_{1} \pm W_{2} \pm W_{3} .
$$

When we do this with the correct initial states of registers 6 and 10 , we will find the expected bias at one of the $2^{3}$ possible sums. The complexity of this point would be, for each $p$ :

$$
2^{2} \times T_{6} \times 8 \times 2^{3}=2^{35},
$$

so $2^{35} \times 2^{27}=2^{62}$. But we can speed up the process: We define two new vectors,

$$
\begin{gathered}
V_{6}\left[k^{\prime}\right]=V_{6, j}[k] \\
V_{10}\left[k^{\prime}\right]=V_{10, j}[k]+c t
\end{gathered}
$$

where $k^{\prime}=j T_{6}-1+k, k^{\prime} \in\left\{0, \ldots, 4 T_{6}-1\right\}$, and $c t=0$ if $k \leq T^{\prime}$ and $c t=0.5$ if $k>T^{\prime}$.

With those two vectors we are going to compute:

$$
\sum_{k^{\prime}=0}^{4 T_{6}-1}(-1)^{V_{6}\left[k^{\prime}+p\right]}\left(V_{10}[k]-\frac{3}{2}\right)+4 \times\left(T^{\prime} \times 1.5+\left(T_{6}-T^{\prime}\right) \times 1\right) .
$$

The issue is now to find the $p$ that maximizes this sum, this is the same as computing the maximum of the crosscorrelation of two sequences of length $4 T_{6}$. We can do that efficiently using a fast Fourier transform as explained in [1, pages 306-312]. The final complexity for computing this sum will be in $4 T_{6} \log _{2}\left(4 T_{6}\right)$. We have not finished yet, as we have to do this for each one of the possible relative states of the $z_{i}$, and we can do that by recomputing $V_{6}\left[k^{\prime}\right]$, which will have a low complexity as all we have to do is to xor the bits of the states corresponding to the $i=j$ when $z_{i} \neq z_{0}$. Thus, the total complexity of this state will be $2^{3} \times 4 T_{6} \log _{2}\left(4 T_{6}\right) \approx 2^{37}$

The time complexity is going to be, finally:

$$
2^{L_{10-1}} \times\left[2^{33.69}+4 T_{6} \log _{2} 4 T_{6} \times 2^{3}\right]+T_{6} \times 2^{2}=2^{67} .
$$

The number of keystream bits that we need is

$$
2^{28.29} \times T_{1}+T_{3} T_{7}+T_{4} T_{5}=2^{50.29}+2^{48.1}+2^{51}<2^{52} .
$$

\subsection{Distinguishing attack against Achterbahn-128}

Now, we present a distinguishing attack against the 128-bit version of Achterbahn which also recovers the initial states of two registers.

We consider the following approximation of the combining function $F$ :

$$
\ell\left(x_{0}, \ldots, x_{12}\right)=x_{0}+x_{1}+x_{2}+x_{3}+x_{4}+x_{7}+x_{8}+x_{9}+x_{10} .
$$

Then, for $\ell(t)=x_{0}(t)+x_{1}(t)+x_{2}(t)+x_{3}(t)+x_{4}(t)+x_{7}(t)+x_{8}(t)+x_{9}(t)+x_{10}(t)$, we have $\operatorname{Pr}[S(t)=\ell(t)]=\frac{1}{2}\left(1+2^{-3}\right)$. 
Parity-checks. If we build a parity check as follows:

$$
\ell \ell \ell(t)=\sum_{\tau \in\left\langle T_{3,8}, T_{1,10}, T_{2,9}\right\rangle} \ell(t+\tau),
$$

the terms containing the sequences $x_{1}, x_{2}, x_{3}, x_{8}, x_{9}, x_{10}$ will disappear from $\ell \ell \ell(t)$, so $\ell \ell \ell(t)$ depends exclusively on the sequences $x_{0}, x_{4}$ and $x_{7}$ :

$$
\begin{aligned}
\ell \ell \ell(t) & =\sum_{\tau \in\left\langle T_{3,8}, T_{1,10}, T_{2,9}\right\rangle} \ell(t+\tau) \\
& =\sum_{\tau \in\left\langle T_{3,8}, T_{1,10}, T_{2,9}\right\rangle} x_{0}(t+\tau)+x_{4}(t+\tau)+x_{7}(t+\tau) \\
& =\sigma_{0}(t)+\sigma_{4}(t)+\sigma_{7}(t),
\end{aligned}
$$

where $\sigma_{0}(t), \sigma_{4}(t)$ and $\sigma_{7}(t)$ are the parity-checks calculated on the sequences generated by NLFSRs 0,4 and 7 .

Adding eight times the approximation has the effect of multiplying the bias eight times, so the bias of

$$
\sigma(t)=\sum_{\tau \in\left\langle T_{3,8}, T_{1,10}, T_{2,9}\right\rangle} S(t+\tau)
$$

where $(S(t))_{t \geq 0}$ is the keystream, is $2^{-8 \times 3}$. So:

$$
\operatorname{Pr}\left[\sigma(t)+\sigma_{0}(t)+\sigma_{4}(t)+\sigma_{7}(t)=1\right]=\frac{1}{2}\left(1-\varepsilon^{8}\right) .
$$

This means that we need $2^{3 \times 8 \times 2} \times 2 \times(74-3) \times \ln (2)=2^{54.63}$ values of $\sigma(t)+$ $\sigma_{0}(t)+\sigma_{4}(t)+\sigma_{7}(t)$ to detect this bias, when we perform an exhaustive search on registers 0,4 and 7 .

We are going to use the algorithm proposed previously for the attack of Achterbahn-128 for computing the sum $\sigma(t)+\sigma_{0}(t)+\sigma_{4}(t)+\sigma_{7}(t)$ over all values of $t$. This algorithm has a lower complexity than an exhaustive search for the initial states of the registers 0,4 and 7 simultaneously. We are going to use it considering register 0 and register 4 together.

The complexity is going to be, finally:

$$
2^{L_{0}-1} \times 2^{L_{4}-1} \times\left[2^{54.63} \times\left(2^{4}+2^{4.7}\right)+O\left(T_{7} \log T_{7}\right)\right]+T_{7} \times 2^{3}=2^{104} .
$$

The length of keystream needed is:

$$
2^{54.63}+T_{1,10}+T_{2,9}+T_{3,8}=2^{54.63}+2^{53}+2^{53}+2^{53}<2^{56} \text { bits. }
$$

\section{Recovering the key}

As explained in the previous attacks, with a variant of a meet-in-the-middle attack we can recover the key once we have found the initial state of some 
registers, and the complexity is going to be smaller than the one needed to perform the previously described distinguishing attack that we need to get the initial state of several registers. So the complexity of the total key-recovery attack is going to be the same one as for the distinguishing attacks.

\section{Conclusion}

We have proposed an attack against Achterbahn- 80 in $2^{67}$ where fewer than $2^{52}$ bits are needed. An attack against Achterbahn-128 is also proposed in $2^{104}$ where fewer than $2^{56}$ bits of keystream are required. After that we can recover the key of Achterbahn- 80 with a complexity of $2^{40}$ in time and $2^{41}$ in memory (the time complexity is less than for the distinguishing part of the attack). For Achterbahn-128 we can recover the key with a complexity of $2^{73}$ in time and $2^{48}$ in memory.

\section{References}

1. R. E. Blahut. Fast Algorithms for Digital Signal Processing. Addison Wesley, 1985.

2. B. M. Gammel, R. Gottfert, and O. Kniffler. The Achterbahn stream cipher. eSTREAM, ECRYPT Stream Cipher Project, Report 2005/002, 2005. http: //www.ecrypt.eu.org/stream/ciphers/achterbahn/achterbahn.pdf.

3. B. M. Gammel, R. Gottfert, and O. Kniffler. Achterbahn-128/80. eSTREAM, ECRYPT Stream Cipher Project, Report 2006/001, 2006. http://www. ecrypt .eu . org/stream/p2ciphers/achterbahn/achterbahn_p2.pdf.

4. B. M. Gammel, R. Gottfert, and O. Kniffler. Status of Achterbahn and tweaks. eSTREAM, ECRYPT Stream Cipher Project, Report 2006/027, 2006. http:// www .ecrypt.eu.org/stream/papersdir/2006/027.pdf.

5. B. M. Gammel, R. Gottfert, and O. Kniffler. Achterbahn-128/80: Design and analysis. In ECRYPT Network of Excellence - SASC Workshop Record, pages 152-165, 2007.

6. M. Hell and T. Johansson. Cryptanalysis of Achterbahn-128/80. eSTREAM, ECRYPT Stream Cipher Project, Report 2006/054, 2006. http://www.ecrypt. eu.org/stream/papersdir/2006/054.pdf.

7. M. Hell and T. Johansson. Cryptanalysis of Achterbahn-version 2. eSTREAM, ECRYPT Stream Cipher Project, Report 2006/042, 2006. http://www. ecrypt.eu . org/stream/ciphers/achterbahn/achterbahn.pdf.

8. T. Johansson, W. Meier, and F. Muller. Cryptanalysis of Achterbahn. In Advances in Cryptology - FSE 2006, volume 4047 of Lecture Notes in Computer Science, pages 1-14. Springer, 2006.

9. M. Naya-Plasencia. Cryptanalysis of Achterbahn-128/80. eSTREAM, ECRYPT Stream Cipher Project, Report 2006/055, 2006. http://www.ecrypt.eu.org/ stream/papersdir/2006/055.pdf. 